\title{
Medidas da precisão experimental em ensaios com genótipos de feijão e de soja
}

\author{
Alberto Cargnelutti Filho(1), Lindolfo Storck ${ }^{(1)}$ e Nerinéia Dalfollo Ribeiro(1)
} (1)Universidade Federal de Santa Maria, Departamento de Fitotecnia, Avenida Roraima no 1.000, Bairro Camobi, CEP 97105-900 Santa Maria,
RS. E-mail: cargnelutti@pq.cnpq.br, lindolfo@pq.cnpq.br, neiadr@smail.ufsm.br

Resumo - O objetivo deste trabalho foi avaliar a adequação das estatísticas herdabilidade, coeficiente de determinação, valor do teste $\mathrm{F}$ para genótipo, índice de diferenciação de Fasoulas e acurácia seletiva, como medidas do grau de precisão experimental em conjuntos de ensaios de competição de genótipos de feijão (Phaseolus vulgaris) e de soja (Glycine max). Foram usados os dados de produtividade de grãos de 72 e 216 ensaios de competição de genótipos de feijão e de soja, respectivamente, realizados no Estado do Rio Grande do Sul. Com base em 12 estatísticas, estimadas para cada ensaio, foram realizadas análises de correlação, de trilha e de agrupamento, e foi calculada a frequência de ensaios em cada classe de precisão experimental. As estatísticas acurácia seletiva, herdabilidade, coeficiente de determinação e valor do teste F para genótipo são mais adequadas do que o coeficiente de variação e a diferença mínima significativa pelo teste de Tukey, em percentagem da média, para se avaliar a precisão experimental, em ensaios de competição de genótipos de feijão e de soja. Por essas estatísticas, $88,89 \%$ dos ensaios de competição de genótipos de feijão e $75 \%$ dos ensaios de competição de genótipos de soja apresentaram precisão alta ou muito alta. Contudo, 2,78 e 10,19\% dos ensaios de competição de genótipos de feijão e de soja, respectivamente, poderiam ser descartados, em razão da insuficiência na precisão experimental.

Termos para indexação: Glycine max, Phaseolus vulgaris, controle de qualidade, ensaios de competição, planejamento experimental, produtividade de grãos.

\section{Measures of experimental precision in common bean and soybean genotype trials}

\begin{abstract}
The objective of this work was to evaluate the appropriateness of statistics heritability, determination coefficient, F-test value for genotype, Fasoulas index of differentiation and selective accuracy, as measures of the degree of experimental precision in a set of common bean (Phaseolus vulgaris) and soybean (Glycine max) genotype competition trials. Grain yield data from 72 genotype competition trials of common bean and 216 of soybean, carried out in Rio Grande do Sul state, Brazil, were used. Based on 12 statistics estimated for each trial, correlation, path and cluster analyses were done, and the trial frequency in each class of experimental precision was calculated. The statistics selective accuracy, heritability, coefficient of determination and F-test value for genotype are more adequate than the coefficient of variation and the significant minimum difference among cultivars by Tukey's test, expressed in percentage of the mean, to evaluate the experimental precision of common bean and soybean yield trials. According to those statistics, $88.89 \%$ of the common bean experiments and $75 \%$ of the soybean experiments showed a high or very high degree of precision, and $2.78 \%$ and $10.19 \%$ of the experiments of common bean and soybean, respectively, could be discarded due to insufficient experimental precision.
\end{abstract}

Index terms: Glycine max, Phaseolus vulgaris, quality control, competition trials, experimental planning, grain yield.

\section{Introdução}

Ensaios capazes de evidenciar as diferenças genéticas entre os genótipos em avaliação são importantes para identificar os materiais promissores e os que devem ser descartados em um programa de melhoramento genético de plantas. Estatísticas como o coeficiente de variação (Pimentel-Gomes, 1990), o índice de variação (Pimentel-Gomes, 1991) ou, como definido posteriormente, o coeficiente de precisão (Storck et al., 2006) e a diferença mínima significativa (DMS) pelo teste de Tukey, em percentagem da média (Lúcio et al., 1999), têm sido utilizadas para essa finalidade. Maiores

Pesq. agropec. bras., Brasília, v.44, n.10, p.1225-1231, out. 2009 
escores dessas estatísticas indicam menor precisão experimental (Storck et al., 2006).

$\mathrm{O}$ coeficiente de variação $(\mathrm{CV})$ é levado em consideração nos ensaios de valor de cultivo e uso, para o registro de cultivares junto ao Serviço Nacional de Proteção de Cultivares, do Ministério da Agricultura. Nos ensaios com feijão, o CV deve ser inferior ou igual a $25 \%$, ou o valor do teste $\mathrm{F}$ para genótipo deve ser significativo a $5 \%$ de probabilidade (Brasil, 2006), para que o ensaio possa ser aproveitado. Nos ensaios com soja, o CV deve ser no máximo de 20\% (Brasil, 1998). No entanto, outras estatísticas como herdabilidade, coeficiente de determinação, valor do teste $\mathrm{F}$ para genótipo, índice de diferenciação de Fasoulas (Fasoulas, 1983) e a acurácia seletiva têm sido propostas (Resende, 2002; Cargnelutti Filho \& Storck, 2007, 2009; Resende \& Duarte, 2007) para avaliar a precisão experimental. Essas estatísticas, por estarem associadas a maiores variabilidades genéticas e a menores variâncias residuais, são mais adequadas que o coeficiente de variação e a diferença mínima significativa (DMS) pelo teste de Tukey, em percentagem da média, para se avaliar a precisão experimental, em ensaios de competição de cultivares de milho (Cargnelutti Filho \& Storck, 2009).

Faixas de precisão experimental com base na acurácia seletiva e, consequentemente, em valores do teste $\mathrm{F}$ para genótipo, foram estabelecidas teoricamente por Resende \& Duarte (2007), e as relações diretas e não lineares de acurácia seletiva com as estatísticas herdabilidade, coeficiente de determinação e valor do teste $\mathrm{F}$ para genótipo foram demonstradas por Cargnelutti Filho \& Storck (2009). Isso permitiu estabelecer limites de classe adequados, a partir dessas estatísticas, para avaliar a precisão experimental de ensaios de competição de cultivares de milho.

As estatísticas herdabilidade, coeficiente de determinação, valor do teste $\mathrm{F}$ para genótipo, índice de diferenciação de Fasoulas e acurácia seletiva, no entanto, ainda não foram avaliadas em conjuntos de ensaios de genótipos de feijão e de soja. Além disso, a precisão experimental dos ensaios de competição de genótipos de feijão e dos ensaios de competição de genótipos de soja, por essas estatísticas, ainda é desconhecida.

O objetivo deste trabalho foi avaliar a adequação das estatísticas herdabilidade, coeficiente de determinação, valor do teste $\mathrm{F}$ para genótipo, índice de diferenciação de Fasoulas e acurácia seletiva, como medidas do grau de precisão experimental, em ensaios de competição de genótipos de feijão e de soja, considerando-se a variável produtividade de grãos.

\section{Material e Métodos}

Foram usados os dados de produtividade de grãos de 72 ensaios de competição de genótipos de feijão e de 216 de soja, realizados no Estado do Rio Grande do Sul. Os ensaios de feijão foram realizados de 1998 a 2008, e os de soja nos anos agrícolas 2003/2004, $2004 / 2005$ e 2005/2006. Os ensaios de feijão e de soja foram executados em delineamento de blocos completos ao acaso. O número de repetições variou entre três e quatro, e o número de genótipos oscilou entre oito e 26, nos ensaios com feijão, e entre 10 e 20 nos ensaios com soja.

Inicialmente, foram feitas as análises de variância para cada um dos ensaios de feijão e de soja realizados, e foram anotadas as seguintes estatísticas: quadrado médio de bloco $\left(\mathrm{QM}_{\mathrm{B}}\right)$, quadrado médio de genótipo $\left(\mathrm{QM}_{\mathrm{G}}\right)$, quadrado médio do erro $\left(\mathrm{QM}_{\mathrm{E}}\right)$, valor do teste $\mathrm{F}$ para genótipo $\left(\mathrm{Fc}=\mathrm{QM}_{\mathrm{G}} / \mathrm{QM}_{\mathrm{E}}\right)$, média geral do ensaio ( $\hat{m})$, coeficiente de variação $\left[\mathrm{CV}=100\left(\mathrm{QM}_{\mathrm{E}}\right)^{0,5} / \hat{\mathrm{m}}\right]$ e herdabilidade média conforme Cruz (2006), $\mathrm{h}^{2}=\left(\mathrm{QM}_{\mathrm{G}}-\mathrm{QM}_{\mathrm{E}}\right) / \mathrm{QM}_{\mathrm{G}}$.

Em seguida, estimou-se, para cada ensaio, a diferença mínima significativa (DMS) entre as médias de cultivares, pelo teste de Tukey, a 5\% de probabilidade, expresso em percentagem da média, por meio da expressão: DMS $=100 \Delta / \hat{\mathrm{m}}$, em que: $\Delta=\mathrm{q}_{\alpha\left(\mathrm{n} ; \mathrm{GL}_{\mathrm{E}}\right)}\left(\mathrm{QM}_{\mathrm{E}} / \mathrm{J}\right)^{0,5}$ e $\mathrm{q}_{\alpha\left(\mathrm{n} ; \mathrm{GL}_{\mathrm{E}}\right)}$ é o valor crítico para o uso do teste de Tukey; n é o número de cultivares; e $\mathrm{GL}_{\mathrm{E}}$ é o número de graus de liberdade do erro. Determinaram-se, ainda, os valores das estatísticas coeficiente de determinação $\left(\mathrm{R}^{2}\right)$, calculada pela expressão $\mathrm{R}^{2}=\mathrm{QM}_{\mathrm{G}} /\left(\mathrm{QM}_{\mathrm{G}}+\mathrm{QM}_{\mathrm{E}}\right)$, e a amplitude das médias $(\mathrm{H})$, estimada pela diferença entre as médias das cultivares com maior e menor produtividades.

O índice de diferenciação de Fasoulas (IF) (Fasoulas, 1983) foi estimado pela expressão IF $=200 \sum_{i=1}^{n} m_{i} /[n(n-1)]$, em que: $n$ é o número total de cultivares e $\mathrm{m}_{\mathrm{i}} \mathrm{o}$ número de médias de cultivares que a i-ésima cultivar supera estatisticamente, após a aplicação do teste de Tukey. Esse índice fornece o percentual de diferenças significativas entre as médias das cultivares que o teste de comparação múltipla consegue detectar. Em seguida, estimou-se a acurácia 
seletiva (AS) (Resende \& Duarte, 2007), por meio das expressões: $\mathrm{AS}=(1-1 / \mathrm{Fc})^{0,5}$, para $\mathrm{Fc} \geq 1$; e $\mathrm{AS}=0$, para $\mathrm{Fc}<1$, em que Fc é o valor do teste $\mathrm{F}$ para genótipo.

Dessa forma, foram obtidas 12 estatísticas $\left(\mathrm{QM}_{\mathrm{B}}\right.$, $\mathrm{QM}_{\mathrm{G}}, \mathrm{QM}_{\mathrm{E}}, \mathrm{Fc}, \hat{\mathrm{m}}, \mathrm{CV}, \mathrm{h}^{2}, \mathrm{DMS}, \mathrm{R}^{2}, \mathrm{H}, \mathrm{IF}$ e AS) para cada ensaio. A partir de então, em cada conjunto de ensaios (72 ensaios de feijão e 216 ensaios de soja), fez-se a análise de correlação linear de Pearson entre as estatísticas, e sua significância foi verificada por meio do teste t de Student, a 5\% de probabilidade. A seguir, realizaram-se o diagnóstico de multicolinearidade (Cruz \& Carneiro, 2003; Cruz, 2006) e a análise de trilha das variáveis principais, candidatas a medidas de precisão experimental dos ensaios (CV, DMS, $\mathrm{h}^{2}, \mathrm{R}^{2}, \mathrm{Fc}$, IF e AS), em função das variáveis explicativas $\left(\mathrm{QM}_{\mathrm{B}}, \mathrm{QM}_{\mathrm{G}}, \mathrm{QM}_{\mathrm{E}}, \hat{\mathrm{m}} \mathrm{e} \mathrm{H}\right)$.

Para cada conjunto de ensaios, determinou-se a matriz de distância euclidiana padronizada entre as estatísticas $\mathrm{QM}_{\mathrm{B}}, \mathrm{QM}_{\mathrm{G}}, \mathrm{QM}_{\mathrm{E}}, \mathrm{Fc}, \hat{\mathrm{m}}, \mathrm{CV}, \mathrm{h}^{2}, \mathrm{DMS}$, $\mathrm{R}^{2}, \mathrm{H}$, IF e AS. Essa matriz, em escala relativa, foi utilizada como medida de dissimilaridade para a análise de agrupamento das estatísticas, pelo método hierárquico de Ward (Cruz \& Carneiro, 2003; Cruz, 2006). Utilizou-se, em seguida, os limites das classes de precisão experimental, estabelecidos em Cargnelutti Filho \& Storck (2009), para calcular as frequências simples e relativas dos ensaios de feijão e de soja, em cada classe. As análises e as estimativas foram realizadas com o auxílio do programa Genes (Cruz, 2006).

\section{Resultados e Discussão}

Em relação aos dados de produtividade de grãos dos 72 ensaios de competição de genótipos de feijão, o diagnóstico de multicolinearidade mostrou que o número de condição oscilou entre 35 [IF em função de $\left(\mathrm{QM}_{\mathrm{B}}, \mathrm{QM}_{\mathrm{G}}, \mathrm{QM}_{\mathrm{E}}\right.$, $\hat{\mathrm{m}}$ e $\left.\left.\mathrm{H}\right)\right]$ e 48 [CV em função de $\left(\mathrm{QM}_{\mathrm{B}}, \mathrm{QM}_{\mathrm{G}}, \mathrm{QM}_{\mathrm{E}}\right.$, $\left.\left.\hat{\mathrm{m}} \mathrm{e} \mathrm{H}\right)\right]$ (Tabela 1). Em relação aos dados de produtividade de grãos dos 216 ensaios de competição de genótipos de soja, o número de condição oscilou entre 24 [Fc e IF em função de $\left(\mathrm{QM}_{\mathrm{B}}, \mathrm{QM}_{\mathrm{G}}, \mathrm{QM}_{\mathrm{E}}\right.$, $\hat{\mathrm{m}}$ e H)] e $36[\mathrm{CV}$ em função de $\left(\mathrm{QM}_{\mathrm{B}}, \mathrm{QM}_{\mathrm{G}}, \mathrm{QM}_{\mathrm{E}}\right.$, $\hat{\mathrm{m}}$ e H)] (Tabela 2). Portanto, a matriz de correlações é classificada como de colinearidade baixa, o que permite a realização adequada da análise de trilha (Cruz \& Carneiro, 2003; Cruz, 2006). Assim, foram realizadas as análises de trilha das variáveis principais $\left(\mathrm{CV}, \mathrm{DMS}, \mathrm{h}^{2}, \mathrm{R}^{2}, \mathrm{Fc}, \mathrm{IF}\right.$ eAS), em função das variáveis explicativas $\mathrm{QM}_{\mathrm{B}}, \mathrm{QM}_{\mathrm{G}}$, $\mathrm{QM}_{\mathrm{E}}, \hat{\mathrm{m}}$ e $\mathrm{H}$, para o conjunto de ensaios de competição de genótipos de feijão e de soja (Tabelas 1 e 2).

De maneira geral, os resultados da análise de trilha foram similares entre o conjunto de 72 ensaios de feijão e o de 216 ensaios de soja (Tabelas 1 e 2), e ainda concordantes com os obtidos por Cargnelutti Filho \& Storck $(2007,2009)$, em relação à produtividade de grãos de um conjunto de 101 ensaios de competição de cultivares de milho.

Os coeficientes de correlação linear de Pearson (r) entre a variável explicativa quadrado médio de bloco $\left(\mathrm{QM}_{\mathrm{B}}\right)$ e as variáveis principais - coeficiente de variação (CV), diferença mínima significativa (DMS) pelo teste de Tukey, em percentagem da média, herdabilidade $\left(\mathrm{h}^{2}\right)$, coeficiente de determinação $\left(\mathrm{R}^{2}\right)$, valor do teste $\mathrm{F}$ para genótipo $(\mathrm{Fc})$, índice de diferenciação de Fasoulas (IF) e acurácia seletiva (AS) - oscilaram entre $r=0,1854\left(\mathrm{QM}_{\mathrm{B}}\right.$ vs. $\left.\mathrm{CV}\right)$ e $\mathrm{r}=-0,2711\left(\mathrm{QM}_{\mathrm{B}}\right.$ vs. $\mathrm{R}^{2}$ ), no conjunto de ensaios de feijão (Tabela 1 ), e entre $r=0,0370\left(Q_{B}\right.$ vs. DMS $)$ e $r=-0,1140\left(Q_{B}\right.$ vs. Fc), no conjunto de ensaios de soja (Tabela 2), com efeitos diretos baixos (Tabelas 1 e 2). Apesar de não haver relação de causa e efeito do $\mathrm{QM}_{\mathrm{B}}$ com essas variáveis principais, há uma separação em dois grupos de variáveis principais. Num primeiro grupo, estão o $\mathrm{CV}$ e a DMS, que apresentaram valores positivos de correlação com o $\mathrm{QM}_{\mathrm{B}}$ e efeitos indiretos negativos, via média, e positivos, via $\mathrm{QM}_{\mathrm{E}}$. Num segundo grupo, as estatísticas herdabilidade, coeficiente de determinação, valor do teste $\mathrm{F}$ para genótipo, índice de diferenciação de Fasoulas e acurácia seletiva apresentaram valores negativos de correlação com o $\mathrm{QM}_{\mathrm{B}}$ e efeito indireto negativo via $\mathrm{QM}_{\mathrm{E}}$. A presença desses efeitos indiretos explica a ausência de relação de causa e efeito do $\mathrm{QM}_{\mathrm{B}}$ sobre o CV, DMS, $\mathrm{h}^{2}, \mathrm{R}^{2}, \mathrm{Fc}$, IF e AS. Portanto, a classificação da precisão experimental por qualquer uma dessas estatísticas é independente do $\mathrm{QM}_{\mathrm{B}}$.

Não houve associação linear entre o $\mathrm{QM}_{\mathrm{G}}$ e o CV $(r=-0,0626$ e $r=0,0469)$ e entre o $Q_{G}$ e a DMS $(\mathrm{r}=-0,0275$ e $\mathrm{r}=0,0542)$, e houve efeitos diretos desprezíveis do $\mathrm{QM}_{\mathrm{G}}$ sobre o CV $(0,0539$ e -0,0043) e DMS (-0,1245 e -0,1198), respectivamente para o conjunto de ensaios de feijão e de soja (Tabelas 1 e 2). Assim, não há associação entre a variabilidade genética $\left(\mathrm{QM}_{\mathrm{G}}\right)$ e o CV e a DMS, ou seja, a classificação da precisão experimental por essas estatísticas independe da variabilidade genética. 
Contudo, houve associação linear positiva entre o $\mathrm{QM}_{\mathrm{G}}(\mathrm{r} \geq 0,3327)$ e as estatísticas $\mathrm{h}^{2}, \mathrm{R}^{2}, \mathrm{Fc}$, IF e AS (Tabelas 1 e 2). Os efeitos diretos do $\mathrm{QM}_{\mathrm{G}}$ foram de mesmo sinal e magnitudes semelhantes em relação à $\mathrm{h}^{2}$ $(0,2094$ e 0,3760$)$, ao $R^{2}(0,3246$ e 0,4511$)$ e oAS $(0,1337$ e 0,3086$)$, e foram superiores quanto ao $\mathrm{Fc}(0,8113$ e $0,6062)$ e IF $(0,6833$ e 0,6594$)$, respectivamente, para os ensaios de feijão e de soja. $\mathrm{O}$ menor efeito direto de $\mathrm{QM}_{\mathrm{G}}$ sobre $\mathrm{h}^{2}, \mathrm{R}^{2}$ e $\mathrm{AS}$, em comparação ao $\mathrm{QM}_{\mathrm{G}}$ sobre Fc e IF, pode ser explicado pela presença de maior efeito indireto positivo via amplitude de médias $(\mathrm{H})$, já que o efeito indireto negativo via $\mathrm{QM}_{\mathrm{E}}$ foi de magnitude semelhante, para as estatísticas $\mathrm{h}^{2}, \mathrm{R}^{2}, \mathrm{Fc}$, IF e AS $\left(-0,4392 \leq\right.$ efeito indireto via $\left.\mathrm{QM}_{\mathrm{E}} \leq-0,5505\right)$. Com esses resultados, evidencia-se que maiores escores das estatísticas $h^{2}, R^{2}, F c$, IF e AS estão associados a maiores variâncias genéticas. Assim, experimentos mais precisos (maiores escores dessas estatísticas) são aqueles que apresentam maior variabilidade genética. Resultados similares foram obtidos por Cargnelutti Filho \& Storck $(2007,2009)$, na cultura do milho.

Apesar dos valores baixos dos coeficientes de correlação linear de Pearson, entre o $\mathrm{QM}_{\mathrm{E}}$ e as estatísticas $\mathrm{CV}, \mathrm{DMS}, \mathrm{h}^{2}, \mathrm{R}^{2}, \mathrm{Fc}$, IF e AS, os efeitos diretos foram altos e de mesmo sinal (Tabelas 1 e 2). Assim, os altos efeitos indiretos negativos via média, em relação ao $\mathrm{CV}$ e DMS, os altos efeitos indiretos positivos via amplitude de médias, em relação a $\mathrm{h}^{2}$, ao $\mathrm{R}^{2}$ e a $\mathrm{AS}$ e, ainda, os altos efeitos indiretos positivos via $\mathrm{QM}_{\mathrm{G}}$, em relação ao Fc e ao IF, explicam as magnitudes elevadas do efeito direto do $\mathrm{QM}_{\mathrm{E}}$ sobre essas estatísticas, e as magnitudes inferiores do coeficiente de correlação

Tabela 1. Estimativas dos coeficientes de correlação de Pearson e respectivas estimativas dos efeitos diretos e indiretos das estatísticas quadrado médio de bloco $\left(\mathrm{QM}_{\mathrm{B}}\right)$, quadrado médio de genótipo $\left(\mathrm{QM}_{\mathrm{G}}\right)$, quadrado médio do erro $\left(\mathrm{QM}_{\mathrm{E}}\right)$, média geral do ensaio ( $\hat{\mathrm{m}})$ e amplitude das médias $(\mathrm{H})$, sobre as estatísticas coeficiente de variação $(\mathrm{CV})$, diferença mínima significativa (DMS) pelo teste de Tukey, em percentagem da média, herdabilidade $\left(h^{2}\right)$, coeficiente de determinação $\left(\mathrm{R}^{2}\right)$, valor do teste $\mathrm{F}$ para genótipo (Fc), índice de diferenciação de Fasoulas (IF) e acurácia seletiva (AS), para os dados de produtividade de grãos de 72 ensaios de competição de genótipos de feijão.

\begin{tabular}{|c|c|c|c|c|c|c|c|c|}
\hline \multirow{2}{*}{$\begin{array}{l}\text { Variável } \\
\text { explicativa }\end{array}$} & \multirow[t]{2}{*}{ Efeito } & \multicolumn{7}{|c|}{ Variável principal } \\
\hline & & $\mathrm{CV}$ & DMS & $\mathrm{h}^{2}$ & $\mathrm{R}^{2}$ & $\mathrm{Fc}$ & IF & $\mathrm{AS}$ \\
\hline \multirow[t]{6}{*}{$\mathrm{QM}_{\mathrm{B}}$} & Direto & $-0,0017$ & $-0,0358$ & 0,0736 & 0,0668 & 0,1573 & 0,1268 & 0,0873 \\
\hline & Indireto via $\mathrm{QM}_{\mathrm{G}}$ & 0,0015 & $-0,0034$ & 0,0057 & 0,0089 & 0,0221 & 0,0186 & 0,0036 \\
\hline & Indireto via $\mathrm{QM}_{\mathrm{E}}$ & 0,3452 & 0,3277 & $-0,3927$ & $-0,4216$ & $-0,4516$ & $-0,4298$ & $-0,3661$ \\
\hline & Indireto via $\hat{\mathrm{m}}$ & $-0,1552$ & $-0,1532$ & 0,0060 & 0,0092 & 0,0284 & 0,0363 & 0,0053 \\
\hline & Indireto via $\mathrm{H}$ & $-0,0044$ & 0,0211 & 0,0732 & 0,0656 & 0,0152 & 0,0108 & 0,0761 \\
\hline & Total (Corr. Pearson) & $0,1854^{\mathrm{ns}}$ & $0,1564^{\mathrm{ns}}$ & $-0,2341^{\mathrm{ns}}$ & $-0,2711^{*}$ & $-0,2285^{\mathrm{ns}}$ & $-0,2372^{\mathrm{ns}}$ & $-0,1937^{\mathrm{ns}}$ \\
\hline \multirow[t]{6}{*}{$\overline{\mathrm{QM}_{\mathrm{G}}}$} & Direto & 0,0539 & $-0,1245$ & 0,2094 & 0,3246 & 0,8113 & 0,6833 & 0,1337 \\
\hline & Indireto via $\mathrm{QM}_{\mathrm{B}}$ & 0,0000 & $-0,0010$ & 0,0020 & 0,0018 & 0,0043 & 0,0035 & 0,0024 \\
\hline & Indireto via $\mathrm{QM}_{\mathrm{E}}$ & 0,4207 & 0,3995 & $-0,4786$ & $-0,5138$ & $-0,5505$ & $-0,5239$ & $-0,4462$ \\
\hline & Indireto via $\hat{\mathrm{m}}$ & $-0,4979$ & $-0,4917$ & 0,0192 & 0,0295 & 0,0913 & 0,1165 & 0,0171 \\
\hline & Indireto via $\mathrm{H}$ & $-0,0393$ & 0,1903 & 0,6600 & 0,5909 & 0,1373 & 0,0977 & 0,6862 \\
\hline & Total (Corr. Pearson) & $-0,0626^{\mathrm{ns}}$ & $-0,0275^{\mathrm{ns}}$ & $0,4120^{*}$ & $0,4330^{*}$ & $0,4937^{*}$ & $0,3771^{*}$ & $0,3933^{*}$ \\
\hline \multirow[t]{6}{*}{$\overline{\mathrm{QM}_{\mathrm{E}}}$} & Direto & 0,7793 & 0,7398 & $-0,8864$ & $-0,9516$ & $-1,0195$ & $-0,9702$ & $-0,8263$ \\
\hline & Indireto via $\mathrm{QM}_{\mathrm{B}}$ & $-0,0008$ & $-0,0159$ & 0,0326 & 0,0296 & 0,0697 & 0,0562 & 0,0387 \\
\hline & Indireto via $\mathrm{QM}_{\mathrm{G}}$ & 0,0291 & $-0,0672$ & 0,1130 & 0,1753 & 0,4380 & 0,3689 & 0,0722 \\
\hline & Indireto via $\hat{\mathrm{m}}$ & $-0,5694$ & $-0,5624$ & 0,0220 & 0,0337 & 0,1044 & 0,1332 & 0,0196 \\
\hline & Indireto via $\mathrm{H}$ & $-0,0268$ & 0,1298 & 0,4504 & 0,4032 & 0,0937 & 0,0667 & 0,4682 \\
\hline & Total (Corr. Pearson) & $0,2114^{\mathrm{ns}}$ & $0,2242^{\mathrm{ns}}$ & $-0,2684 *$ & $-0,3098^{*}$ & $-0,3137^{*}$ & $-0,3452 *$ & $-0,2276^{\mathrm{ns}}$ \\
\hline \multirow[t]{6}{*}{$\overline{\mathrm{m}}$} & Direto & $-1,0499$ & $-1,0370$ & 0,0406 & 0,0622 & 0,1925 & 0,2457 & 0,0361 \\
\hline & Indireto via $\mathrm{QM}_{\mathrm{B}}$ & $-0,0003$ & $-0,0053$ & 0,0109 & 0,0099 & 0,0232 & 0,0187 & 0,0129 \\
\hline & Indireto via $\mathrm{QM}_{\mathrm{G}}$ & 0,0256 & $-0,0590$ & 0,0993 & 0,1539 & 0,3847 & 0,3240 & 0,0634 \\
\hline & Indireto via $\mathrm{QM}_{\mathrm{E}}$ & 0,4226 & 0,4012 & $-0,4807$ & $-0,5161$ & $-0,5529$ & $-0,5262$ & $-0,4481$ \\
\hline & Indireto via $\mathrm{H}$ & $-0,0217$ & 0,1051 & 0,3647 & 0,3265 & 0,0759 & 0,0540 & 0,3792 \\
\hline & Total (Corr. Pearson) & $-0,6237 *$ & $-0,5949^{*}$ & $0,0347^{\text {ns }}$ & $0,0365^{\text {ns }}$ & $0,1234^{\mathrm{ns}}$ & $0,1162^{\text {ns }}$ & $0,0435^{\mathrm{ns}}$ \\
\hline \multirow[t]{9}{*}{$\overline{\mathrm{H}}$} & Direto & $-0,0434$ & 0,2100 & 0,7285 & 0,6522 & 0,1516 & 0,1079 & 0,7574 \\
\hline & Indireto via $\mathrm{QM}_{\mathrm{B}}$ & $-0,0002$ & $-0,0036$ & 0,0074 & 0,0067 & 0,0158 & 0,0127 & 0,0088 \\
\hline & Indireto via $\mathrm{QM}_{\mathrm{G}}$ & 0,0489 & $-0,1128$ & 0,1897 & 0,2941 & 0,7350 & 0,6191 & 0,1212 \\
\hline & Indireto via $\mathrm{QM}_{\mathrm{E}}$ & 0,4818 & 0,4574 & $-0,5480$ & $-0,5883$ & $-0,6303$ & $-0,5998$ & $-0,5109$ \\
\hline & Indireto via $\hat{\mathrm{m}}$ & $-0,5257$ & $-0,5191$ & 0,0203 & 0,0311 & 0,0964 & 0,1230 & 0,0181 \\
\hline & Total (Corr. Pearson) & $-0,0386^{\mathrm{ns}}$ & $0,0318^{\mathrm{ns}}$ & $0,3979 *$ & $0,3959 *$ & $0,3685^{*}$ & $0,2628^{*}$ & $0,3945^{*}$ \\
\hline & Coef. de determinação & 0,8176 & 0,7873 & 0,5982 & 0,6777 & 0,7640 & 0,6194 & 0,5242 \\
\hline & Variável residual & 0,4271 & 0,4612 & 0,6339 & 0,5677 & 0,4858 & 0,6169 & 0,6898 \\
\hline & Número de condição & 48 & 42 & 36 & 36 & 44 & 35 & 36 \\
\hline
\end{tabular}

${ }^{n}$ Não significativo. *Significativo a $5 \%$ de probabilidade, pelo teste $\mathrm{t}$, com 70 graus de liberdade. 
de Pearson. Com esses resultados, demonstra-se que menores variâncias residuais estão associadas a menores escores de CV e DMS e a maiores escores de $\mathrm{h}^{2}, \mathrm{R}^{2}, \mathrm{Fc}, \mathrm{IF}$ e AS. Do ponto de vista de classificação da precisão experimental, seriam mais precisos os ensaios com menores escores de CV e DMS ou maiores escores de $\mathrm{h}^{2}, \mathrm{R}^{2}, \mathrm{Fc}$, IF e AS. Os resultados observados por Cargnelutti Filho \& Storck $(2007,2009)$ corroboram essas inferências.

Houve associação linear negativa entre a média e o $\mathrm{CV}(\mathrm{r}=-0,6237$ e $\mathrm{r}=-0,5136)$ e entre a média e a DMS $(r=-0,5949$ e $r=-0,4831)$, com efeitos diretos altos e de mesmo sinal da média sobre o CV (-1,0499 e $-1,0015)$ e DMS (-1,0370 e -0,9783), respectivamente, para os ensaios de feijão e os de soja, o que mostra associação de causa e efeito entre a média e essas estatísticas (Tabelas 1 e 2). No entanto, os valores do coeficiente de correlação linear de Pearson entre a média e as estatísticas $\mathrm{h}^{2}, \mathrm{R}^{2}, \mathrm{Fc}$, IF e AS foram baixos $(\mathrm{r} \leq|0,1890|)$, com efeito direto desprezível da média sobre essas estatísticas. Portanto, o CV e a DMS são dependentes da média, enquanto as estatísticas $h^{2}$, $\mathrm{R}^{2}, \mathrm{Fc}$, IF e AS independem dela, o que lhes confere uma vantagem sobre as primeiras, do ponto de vista de classificação da precisão experimental (Cargnelutti Filho \& Storck, 2007, 2009; Resende \& Duarte, 2007).

Não houve associação linear entre a amplitude de médias $(\mathrm{H})$ e o $\mathrm{CV}(\mathrm{r}=-0,0386$ e $\mathrm{r}=0,0556)$ e $\mathrm{H}$ e a DMS $(r=0,0318$ e $r=0,0944)$, com efeitos diretos baixos de $\mathrm{H}$ sobre o CV $(-0,0434$ e - 0,0349$)$ e sobre a DMS $(0,2100$ e 0,0986$)$, respectivamente, para os ensaios

Tabela 2. Estimativas dos coeficientes de correlação de Pearson e respectivas estimativas dos efeitos diretos e indiretos das estatísticas quadrado médio de bloco $\left(\mathrm{QM}_{\mathrm{B}}\right)$, quadrado médio de genótipo $\left(\mathrm{QM}_{\mathrm{G}}\right)$, quadrado médio do erro $\left(\mathrm{QM}_{\mathrm{E}}\right)$, média geral do ensaio ( $\hat{\mathrm{m}})$ e amplitude das médias $(\mathrm{H})$, sobre as estatísticas coeficiente de variação $(\mathrm{CV})$, diferença mínima significativa (DMS) pelo teste de Tukey, em percentagem da média, herdabilidade $\left(\mathrm{h}^{2}\right)$, coeficiente de determinação $\left(\mathrm{R}^{2}\right)$, valor do teste $\mathrm{F}$ para genótipo $(\mathrm{Fc})$, índice de diferenciação de Fasoulas (IF) e acurácia seletiva (AS), para os dados de produtividade de grãos de 216 ensaios de competição de genótipos de soja.

\begin{tabular}{|c|c|c|c|c|c|c|c|c|}
\hline \multirow{2}{*}{$\begin{array}{l}\text { Variável } \\
\text { explicativa }\end{array}$} & \multirow[t]{2}{*}{ Efeito } & \multicolumn{7}{|c|}{ Variável principal } \\
\hline & & $\mathrm{CV}$ & DMS & $h^{2}$ & $\mathrm{R}^{2}$ & $\mathrm{Fc}$ & IF & $\mathrm{AS}$ \\
\hline \multirow[t]{6}{*}{$\mathrm{QM}_{\mathrm{B}}$} & Direto & 0,0412 & 0,0384 & $-0,0043$ & $-0,0245$ & $-0,0560$ & $-0,0608$ & 0,0158 \\
\hline & Indireto via $\mathrm{QM}_{\mathrm{G}}$ & $-0,0004$ & $-0,0123$ & 0,0386 & 0,0463 & 0,0623 & 0,0677 & 0,0317 \\
\hline & Indireto via $\mathrm{QM}_{\mathrm{E}}$ & 0,1436 & 0,1428 & $-0,1569$ & $-0,1617$ & $-0,1456$ & $-0,1360$ & $-0,1470$ \\
\hline & Indireto via $\hat{\mathrm{m}}$ & $-0,1437$ & $-0,1404$ & $-0,0019$ & $-0,0008$ & 0,0070 & 0,0057 & $-0,0027$ \\
\hline & Indireto via $\mathrm{H}$ & $-0,0030$ & 0,0086 & 0,0481 & 0,0434 & 0,0183 & 0,0147 & 0,0501 \\
\hline & Total (Corr. Pearson) & $0,0376^{\mathrm{ns}}$ & $0,0370^{\mathrm{ns}}$ & $-0,0763^{\mathrm{ns}}$ & $-0,0972^{\mathrm{ns}}$ & $-0,1140^{\mathrm{ns}}$ & $-0,1087^{\mathrm{ns}}$ & $-0,0522^{\mathrm{ns}}$ \\
\hline \multirow[t]{6}{*}{$\overline{\mathrm{QM}_{\mathrm{G}}}$} & Direto & $-0,0043$ & $-0,1198$ & 0,3760 & 0,4511 & 0,6062 & 0,6594 & 0,3086 \\
\hline & Indireto via $\mathrm{QM}_{\mathrm{B}}$ & 0,0042 & 0,0039 & $-0,0004$ & $-0,0025$ & $-0,0058$ & $-0,0062$ & 0,0016 \\
\hline & Indireto via $\mathrm{QM}_{\mathrm{E}}$ & 0,4638 & 0,4610 & $-0,5066$ & $-0,5223$ & $-0,4701$ & $-0,4392$ & $-0,4748$ \\
\hline & Indireto via $\hat{\mathrm{m}}$ & $-0,3863$ & $-0,3773$ & $-0,0051$ & $-0,0021$ & 0,0189 & 0,0152 & $-0,0072$ \\
\hline & Indireto via $\mathrm{H}$ & $-0,0306$ & 0,0863 & 0,4852 & 0,4377 & 0,1847 & 0,1482 & 0,5045 \\
\hline & Total (Corr. Pearson) & $0,0469^{\text {ns }}$ & $0,0542^{\mathrm{ns}}$ & $0,3490 *$ & $0,3619^{*}$ & $0,3340^{*}$ & $0,3774 *$ & $0,3327^{*}$ \\
\hline \multirow[t]{6}{*}{$\overline{\mathrm{QM}_{\mathrm{E}}}$} & Direto & 0,8712 & 0,8661 & $-0,9517$ & $-0,9812$ & $-0,8831$ & $-0,8251$ & $-0,8920$ \\
\hline & Indireto via $\mathrm{QM}_{\mathrm{B}}$ & 0,0068 & 0,0063 & $-0,0007$ & $-0,0040$ & $-0,0092$ & $-0,0100$ & 0,0026 \\
\hline & Indireto via $\mathrm{QM}_{\mathrm{G}}$ & $-0,0023$ & $-0,0638$ & 0,2002 & 0,2401 & 0,3227 & 0,3510 & 0,1643 \\
\hline & Indireto via $\hat{\mathrm{m}}$ & $-0,5725$ & $-0,5592$ & $-0,0076$ & $-0,0031$ & 0,0280 & 0,0226 & $-0,0107$ \\
\hline & Indireto via $\mathrm{H}$ & $-0,0202$ & 0,0571 & 0,3213 & 0,2898 & 0,1223 & 0,0981 & 0,3341 \\
\hline & Total (Corr. Pearson) & $0,2830 *$ & $0,3065^{*}$ & $-0,4386^{*}$ & $-0,4583^{*}$ & $-0,4193 *$ & $-0,3634^{*}$ & $-0,4017 *$ \\
\hline \multirow[t]{6}{*}{$\hat{\mathrm{m}}$} & Direto & $-1,0015$ & $-0,9783$ & $-0,0133$ & $-0,0054$ & 0,0490 & 0,0395 & $-0,0188$ \\
\hline & Indireto via $\mathrm{QM}_{\mathrm{B}}$ & 0,0059 & 0,0055 & $-0,0006$ & $-0,0035$ & $-0,0080$ & $-0,0087$ & 0,0023 \\
\hline & Indireto via $\mathrm{QM}_{\mathrm{G}}$ & $-0,0017$ & $-0,0462$ & 0,1450 & 0,1740 & 0,2338 & 0,2543 & 0,1190 \\
\hline & Indireto via $\mathrm{QM}_{\mathrm{E}}$ & 0,4980 & 0,4951 & $-0,5441$ & $-0,5609$ & $-0,5048$ & $-0,4716$ & $-0,5099$ \\
\hline & Indireto via $\mathrm{H}$ & $-0,0144$ & 0,0408 & 0,2293 & 0,2068 & 0,0873 & 0,0700 & 0,2384 \\
\hline & Total (Corr. Pearson) & $-0,5136^{*}$ & $-0,4831^{*}$ & $-0,1837^{*}$ & $-0,1890 *$ & $-0,1428^{*}$ & $-0,1165^{\mathrm{ns}}$ & $-0,1690^{*}$ \\
\hline \multirow[t]{9}{*}{$\overline{\mathrm{H}}$} & Direto & $-0,0349$ & 0,0986 & 0,5543 & 0,5000 & 0,2110 & 0,1693 & 0,5763 \\
\hline & Indireto via $\mathrm{QM}_{\mathrm{B}}$ & 0,0036 & 0,0033 & $-0,0004$ & $-0,0021$ & $-0,0049$ & $-0,0053$ & 0,0014 \\
\hline & Indireto via $\mathrm{QM}_{\mathrm{G}}$ & $-0,0038$ & $-0,1048$ & 0,3291 & 0,3949 & 0,5306 & 0,5772 & 0,2702 \\
\hline & Indireto via $\mathrm{QM}_{\mathrm{E}}$ & 0,5050 & 0,5020 & $-0,5517$ & $-0,5687$ & $-0,5119$ & $-0,4782$ & $-0,5170$ \\
\hline & Indireto via $\hat{\mathrm{m}}$ & $-0,4142$ & $-0,4047$ & $-0,0055$ & $-0,0022$ & 0,0203 & 0,0163 & $-0,0078$ \\
\hline & Total (Corr. Pearson) & $0,0556^{\text {ns }}$ & $0,0944^{\text {ns }}$ & $0,3259^{*}$ & $0,3218^{*}$ & $0,2452^{*}$ & $0,2793^{*}$ & $0,3231^{*}$ \\
\hline & Coef. de determinação & 0,7603 & 0,7423 & 0,7320 & 0,7773 & 0,6238 & 0,5979 & 0,6496 \\
\hline & Variável residual & 0,4896 & 0,5076 & 0,5176 & 0,4720 & 0,6133 & 0,6341 & 0,5920 \\
\hline & Número de condição & 36 & 34 & 29 & 34 & 24 & 24 & 25 \\
\hline
\end{tabular}

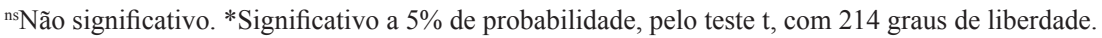


de feijão e de soja (Tabelas 1 e 2). Isso demonstra a independência de $\mathrm{H}$ em relação ao $\mathrm{CV}$ e à DMS. Todavia, houve associação linear positiva entre $\mathrm{H}$ e as estatísticas $h^{2}, R^{2}, F c$, IF e AS ( $\left.r \geq 0,2452\right)$. Os efeitos diretos de mesmo sinal e altos da amplitude de médias (H) sobre $h^{2}, R^{2}$ e AS $(\geq 0,5000)$ mostram que ensaios com maiores amplitudes de médias estão associados a maiores escores de $\mathrm{h}^{2}, \mathrm{R}^{2}$ e AS. Os efeitos diretos de $\mathrm{H}$ sobre $\mathrm{Fc}(0,1516$ e 0,2110$)$ e IF $(0,1079$ e 0,1693$)$ são explicados pelos efeitos indiretos positivos e elevados via $\mathrm{QM}_{\mathrm{G}}$, já que os efeitos indiretos negativos via $\mathrm{QM}_{\mathrm{E}}$ foram de magnitude semelhante para as estatísticas $h^{2}$, $\mathrm{R}^{2}, \mathrm{Fc}$, IF e AS (Tabelas 1 e 2).

De maneira geral, os resultados dos conjuntos de ensaios de feijão e de soja evidenciaram dois grupos de estatísticas. Em um primeiro grupo, estão o CV e a DMS, que apresentaram associação positiva com a variância residual $\left(\mathrm{QM}_{\mathrm{E}}\right)$ e negativa com a média, além de serem independentes do $\mathrm{QM}_{\mathrm{B}}$, do $\mathrm{QM}_{\mathrm{G}}$ e da amplitude. No segundo grupo, estão as estatísticas $\mathrm{h}^{2}, \mathrm{R}^{2}, \mathrm{Fc}$, IF e AS, com associação positiva com a variância genética $\left(\mathrm{QM}_{\mathrm{G}}\right)$, e a amplitude, negativa com a variância residual $\left(\mathrm{QM}_{\mathrm{E}}\right)$, e independentes do $\mathrm{QM}_{\mathrm{B}}$ e da média do ensaio. Esses resultados reforçam a afirmação de Cargnelutti Filho \& Storck (2009), de que as estatísticas acurácia seletiva, herdabilidade, coeficiente de determinação e valor do teste $\mathrm{F}$ para genótipos são mais adequadas do que o CV e a DMS pelo teste de Tukey, em percentagem da média, para se avaliar a precisão experimental.

Em ambos os conjuntos de ensaios, houve a formação de três grupos de estatísticas ao se utilizar $50 \%$ de similaridade como critério para a definição dos grupos, pelo método de Ward (Figura 1). Assim, esses dendrogramas reforçam as discussões anteriores, ou seja, as estatísticas $\mathrm{R}^{2}, \mathrm{AS}, \mathrm{h}^{2}$, IF e Fc, destacadas como adequadas para a classificação da precisão experimental, formaram um grupo, o que comprova serem estatísticas similares, e as estatísticas DMS e $\mathrm{CV}$ formaram outro grupo. Essas últimas estatísticas, por estarem associadas à média e à variância residual, são medidas adequadas de precisão experimental para ensaios com médias semelhantes (Cargnelutti Filho \& Storck, 2007). As variáveis explicativas na análise de trilha (média, $\mathrm{QM}_{\mathrm{E}}, \mathrm{H}, \mathrm{QM}_{\mathrm{G}} \mathrm{e} \mathrm{QM}_{\mathrm{B}}$ ) foram enquadradas em outro grupo. Resultados similares foram verificados por Cargnelutti Filho \& Storck (2009).
Pelas estatísticas analisadas, $88,89 \%$ dos ensaios de competição de genótipos de feijão e $75 \%$ dos de soja apresentaram precisão alta ou muito alta $(\mathrm{AS} \geq 0,70$; $h^{2} \geq 0,49 ; R^{2} \geq 0,6623$ e $\left.F c \geq 1,9608\right)$. Além disso, 2,78\% e $10,19 \%$ dos ensaios de competição de genótipos de feijão e de soja, respectivamente, poderiam ser descartados por apresentar precisão experimental insuficiente (Tabela 3). Com base nessas estatísticas, Cargnelutti Filho \& Storck (2009) concluíram que
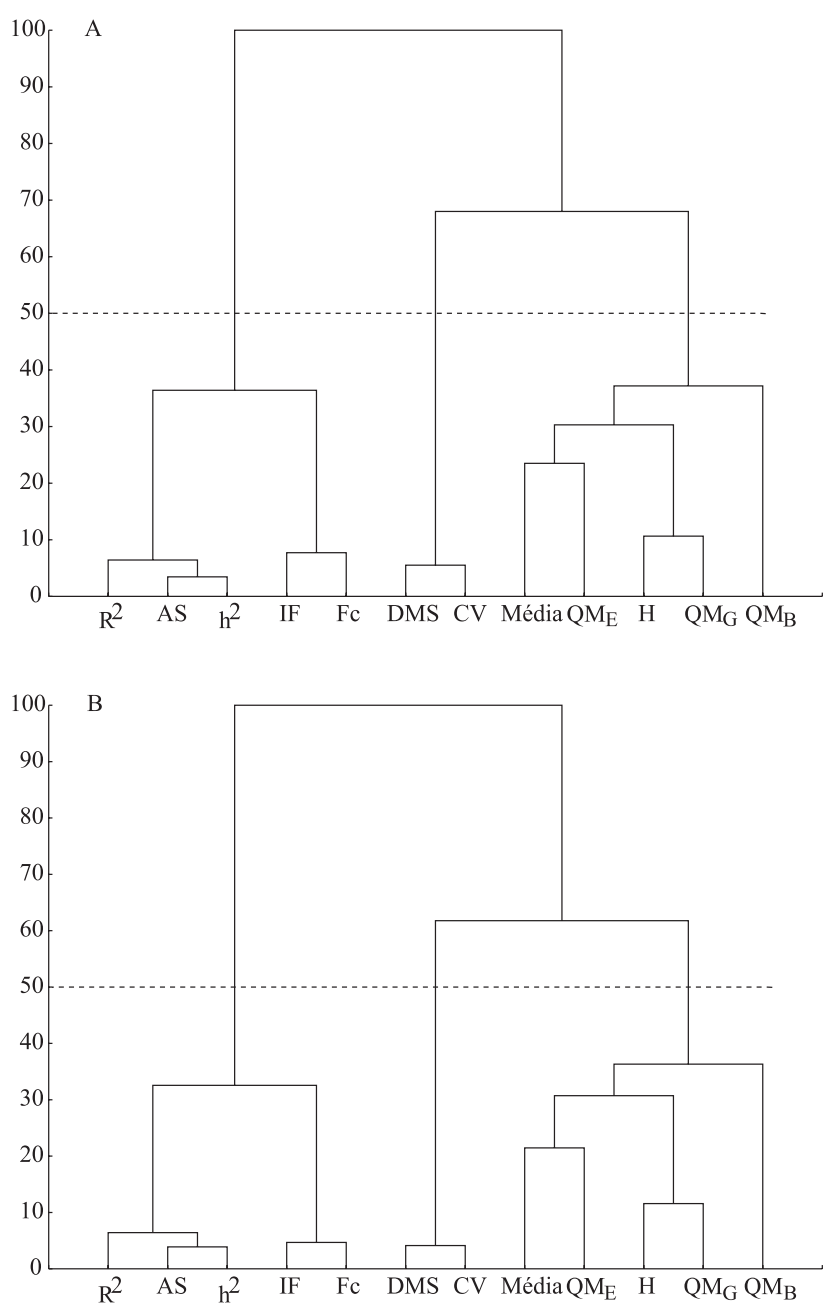

Figura 1. Dendrograma das estatísticas quadrado médio de bloco $\left(\mathrm{QM}_{\mathrm{B}}\right)$, quadrado médio de genótipo $\left(\mathrm{QM}_{\mathrm{G}}\right)$, quadrado médio do erro $\left(\mathrm{QM}_{\mathrm{E}}\right)$, valor do teste $\mathrm{F}$ para genótipo $(\mathrm{Fc})$, média geral do ensaio (média), coeficiente de variação (CV), herdabilidade $\left(\mathrm{h}^{2}\right)$, diferença mínima significativa (DMS) pelo teste de Tukey, em percentagem da média, coeficiente de determinação $\left(\mathrm{R}^{2}\right)$, amplitude das médias $(\mathrm{H})$, índice de diferenciação de Fasoulas (IF) e acurácia seletiva (AS), para os ensaios de feijão (A) e de soja (B). Os dendrogramas foram obtidos pelo método de Ward, a partir da matriz de distância euclidiana padronizada. 
Tabela 3. Limites das classes de precisão experimental (Cargnelutti Filho \& Storck, 2009), em relação à produtividade de grãos, para as estatísticas acurácia seletiva (AS) (Resende \& Duarte, 2007), herdabilidade ( ${ }^{2}$ ) (Cargnelutti Filho \& Storck, 2007), coeficiente de determinação $\left(R^{2}\right)$ (Cargnelutti Filho \& Storck, 2007) e valor do teste F para genótipo (Fc) (Cargnelutti Filho \& Storck, 2007; Resende \& Duarte, 2007), e frequências simples ( $\left.\mathrm{f}_{\mathrm{i}}\right)$ e relativa $\left(\mathrm{fr}_{\mathrm{i}}\right)$ de ensaios de competição de genótipos de feijão e de soja em cada classe.

\begin{tabular}{|c|c|c|c|c|c|c|c|c|}
\hline \multirow{2}{*}{$\begin{array}{l}\text { Precisão } \\
\text { experimental }\end{array}$} & \multirow{2}{*}{$\mathrm{AS}=\sqrt{1-\frac{1}{\mathrm{Fc}}}$} & \multirow{2}{*}{$\mathrm{h}^{2}=\mathrm{AS}^{2}$} & \multirow{2}{*}{$\mathrm{R}^{2}=\frac{1}{2-\mathrm{AS}^{2}}$} & \multirow{2}{*}{$\mathrm{Fc}=\frac{1}{1-\mathrm{AS}^{2}}$} & \multicolumn{2}{|c|}{ Feijão } & \multicolumn{2}{|c|}{ Soja } \\
\hline & & & & & $\mathrm{f}_{\mathrm{i}}$ & $\mathrm{fr}_{\mathrm{i}}(\%)$ & $\mathrm{f}_{\mathrm{i}}$ & $\mathrm{fr}_{\mathrm{i}}(\%)$ \\
\hline Muito Alta & $\geq 0,90$ & $\geq 0,81$ & $\geq 0,8403=$ & $\geq 5,2632$ & 24 & 33,33 & 34 & 15,74 \\
\hline Alta & $\geq 0,70 \mathrm{e}<0,90$ & $\geq 0,49 \mathrm{e}<0,81$ & $\geq 0,6623$ e $<0,8403$ & $\geq 1,9608$ e $<5,2632$ & 40 & 55,56 & 128 & 59,26 \\
\hline Moderada & $\geq 0,50 \mathrm{e}<0,70$ & $\geq 0,25$ e $<0,49$ & $\geq 0,5714$ e $<0,6623$ & $\geq 1,3333$ e $<1,9608$ & 6 & 8,33 & 32 & 14,81 \\
\hline Bälxa & $<0,5$ & $<0,25$ & $<0,5714$ & $<1,3333$ & 2 & 2,78 & 22 & 10,19 \\
\hline
\end{tabular}

$89,10 \%$ de 101 ensaios de competição de cultivares de milho apresentaram precisão alta ou muito alta, e que $4,95 \%$ dos ensaios poderiam ser descartados devido à baixa precisão experimental.

As propriedades favoráveis das estatísticas acurácia seletiva, herdabilidade, coeficiente de determinação e valor do teste $\mathrm{F}$ para genótipo, e as relações entre elas, sustentadas em Cargnelutti Filho \& Storck (2007, 2009) e Resende \& Duarte (2007) e confirmadas neste trabalho, as credenciam como alternativas adequadas para a classificação da precisão experimental de ensaios de competição de genótipos de milho, feijão e soja, com base nos limites estabelecidos em Cargnelutti Filho \& Storck (2009).

\section{Conclusão}

As estatísticas acurácia seletiva, herdabilidade, coeficiente de determinação e valor do teste $\mathrm{F}$ para genótipo são mais adequadas do que o coeficiente de variação e a diferença mínima significativa pelo teste de Tukey, em percentagem da média, para se avaliar a precisão experimental em ensaios de competição de genótipos de feijão e de soja.

\section{Agradecimentos}

Ao Conselho Nacional de Desenvolvimento Científico e Tecnológico, pela concessão de bolsas. Aos pesquisadores das instituições de pesquisa Fundação Estadual de Pesquisa Agropecuária, Empresa Brasileira de Pesquisa Agropecuária, Fundação Centro de Experimentação e Pesquisa e Universidade Federal de Santa Maria, pela cessão dos dados.

\section{Referências}

BRASIL. Ministério da Agricultura, Pecuária e Abastecimento. Requisitos mínimos para determinação do valor de cultivo e uso de feijão (Phaseolus vulgaris), para a inscrição no registro nacional de cultivares - RNC. Brasília: MAPA, 2006. Anexo I.

BRASIL. Ministério da Agricultura, Pecuária e Abastecimento. Requisitos mínimos para determinação do valor de cultivo e uso de soja (Glycine max), para a inscrição no registro nacional de cultivares - RNC. Brasília: MAPA, 1998. Anexo VI.

CARGNELUTTI FILHO, A.; STORCK, L. Estatísticas de avaliação da precisão experimental em ensaios de cultivares de milho. Pesquisa Agropecuária Brasileira, v.42, p.17-24, 2007.

CARGNELUTTI FILHO, A.; STORCK, L. Medidas do grau de precisão experimental em ensaios de competição de cultivares de milho. Pesquisa Agropecuária Brasileira, v.44, p.111-117, 2009.

CRUZ, C.D. Programa GENES: estatística experimental e matrizes. Viçosa: UFV, 2006. 285p.

CRUZ, C.D.; CARNEIRO, P.C.S. Modelos biométricos aplicados ao melhoramento genético. Viçosa: UFV, 2003. v.2, 585p.

FASOULAS, A.C. Rating cultivars and trials in applied plant breeding. Euphytica, v.32, p.939-943, 1983.

LÚCIO, A.D.; STORCK, L.; BANZATTO, D.A. Classificação dos experimentos de competição de cultivares quanto a sua precisão. Pesquisa Agropecuária Gaúcha, v.5, p.99-103, 1999.

PIMENTEL-GOMES, F. Curso de estatística experimental. 13.ed. Piracicaba: Nobel, 1990. 468p.

PIMENTEL-GOMES, F. O índice de variação, um substituto vantajoso do coeficiente de variação. Piracicaba: IPEF, 1991. 4p. (Circular Técnica, 178).

RESENDE, M.D.V. de. Genética biométrica e estatística no melhoramento de plantas perenes. Brasília: Embrapa Informação Tecnológica; Colombo: Embrapa Florestas, 2002. 975p.

RESENDE, M.D.V. de; DUARTE, J.B. Precisão e controle de qualidade em experimentos de avaliação de cultivares. Pesquisa Agropecuária Tropical, v.37, p.182-194, 2007.

STORCK, L.; GARCIA, D.C.; LOPES, S.J.; ESTEFANEL, V. Experimentação vegetal. Santa Maria: UFSM, 2006. 198p.

Recebido em 9 de abril de 2009 e aprovado em 6 de setembro de 2009 